\title{
The prevalence and risk of missing outcome data in prenatal vitamin D supplemented gestational diabetes mellitus patients: a systematic review and meta-analysis protocol
}

\author{
Sumanta Saha ${ }^{1 *}$
}

\begin{abstract}
Background: Missing outcome data in clinical trials are important determinants of internal validity; however, its burden and risk in gestational diabetes mellitus (GDM) mothers supplemented with vitamin D remain poorly studied.

Therefore, a systematic review and meta-analysis protocol is proposed here to study it.

Methods: The English language publications, irrespective of its publication date, will be searched in electronic databases for randomized controlled trials studying the above outcome. The eligible trials will undergo the risk of bias assessment by the Cochrane tool. Data on its trial design, population characteristics, interventions compared, and the outcome will be abstracted. The prevalence and incidence (in risk ratio) of missing outcome data will be estimated meta-analytically. The statistical heterogeneity assessment will include the use of $C h i^{2}$ and $R^{2}$ statistics. For the explanation of any substantial heterogeneity, a meta-regression analysis will ensue. The statistical significance will be determined at $P<0.05$ and $95 \% \mathrm{Cl}$. All analyses will be done in Stata statistical software. If the quantitative analysis is not possible, narrative reporting will happen.
\end{abstract}

Results: The reporting of the review will follow the PRISMA guideline. Statistically significant pairwise meta-analysis finding's grading will occur by the GRADE approach.

Conclusion: The proposed review will estimate the prevalence of missing outcome data in vitamin D supplemented GDM mothers in clinical trials and compare its risk with the placebo recipients.

PROSPERO registration ID: CRD42020180634

Keywords: Gestational Diabetes Mellitus, Prevalence Study, Randomized Controlled Trial, Vitamin D, Epidemiologic Biases, India

\section{Background}

Gestational diabetes mellitus (GDM) is a medical complication of pregnancy. It manifests as glucose intolerance (of any degree) that is detected or developed for the first time during pregnancy [1]. With the rising trend in obesity and a sedentary lifestyle, its prevalence in reproductive age group females is increasing globally [2]. GDM's complications can affect both the mother and her neonate. Maternal complications may include an increased risk of type 2 diabetes development, cesarean section, pre-eclampsia, and polyhydramnios $[1,3,4]$. Some of the fetal complications include newborn

*Correspondence: sumanta.saha@uq.net.au

${ }^{1}$ Independent Researcher, India. ORCID ID: 0000-0003-0996-8846

Full list of author information is available at the end of the article hypoglycemia, hyperbilirubinemia, and increased perinatal mortality [1]. Besides the contemporary GDM management practice by dietary modifications, compliance with selfmonitoring of blood glucose levels, and medications [1,5,6], novel researches are exploring the role of antenatal vitamin D supplementation in the management of GDM and its complications. Recently, several trials have studied the effect of antenatal vitamin D supplementation in GDM mothers. A metaanalysis of such trials found that vitamin D supplementation might improve certain blood glucose and lipid parameters (e.g., homeostatic model assessment for insulin resistance, quantitative insulin sensitivity check index, and low-density lipoprotein cholesterol) [7]. Other systematic reviews reported a reduced risk of cesarean section, macrosomia, neonatal hospitalization, and newborn hyperbilirubinemia in vitamin D supplemented GDM patients [8,9]. 
Amidst of these growing numbers of trials testing the effect of antenatal vitamin D supplementation in GDM mothers, it is crucial to study the validity of its findings due to the missing outcome data. Bias due to the latter can also contaminate the meta-analysis findings that extract data from these trials [8-10]. Notably, attrition is common even in the adequately conducted randomized controlled trials (RCT) [11]. In vitamin D supplemented trials on GDM patients, both the continuous (e.g., gestational weight, body mass index, blood glucose, and lipid parameters) and dichotomous (e.g., the frequency of cesarean section, pre-eclampsia, preterm delivery, neonatal jaundice, and newborn hypoglycemia) outcomes can be affected due to missingness of participants from the trials. For continuous outcomes, the proportion of missing data determines the risk of bias. Among two hypothetical trials with identical observed mean and missing values' average, the mean difference of the outcomes between the compared intervention groups will be higher in the trial with a higher number of participant attrition [12]. Whereas, for dichotomous outcomes, comparing the missing data to the frequency of events ratio across multiple trials helps to juxtapose their risk of bias [12].

Additionally, the causes of missingness between the intervention arms also determine the risk of bias in RCTs [12]. For instance, in two trials investigating the effects of antenatal vitamin D supplementation on GDM patients, the reasons for missingness were not identical between the treatment groups $[13,14]$. While intra-uterine fetal demise, placental abruption, and hospitalization were the causes of missing outcome data in the vitamin D recipients, insulin therapy, pre-eclampsia, hospitalization, and placental abruption were the causes in the comparison group $[13,14]$. Along with this, to minimize the risk of bias, a balance in attrition frequency between the intervention groups of a trial is required [12].

Given these implications of incomplete outcome data, it is imperative to investigate its epidemiological burden in antenatal vitamin D supplemented GDM mothers and how its risk differs from those of placebo recipients.

\section{Intervention description}

Vitamin D is a fat-soluble hormone, available from diet and supplements in two inactive forms: D2 (ergocalciferol) and D3 (cholecalciferol) $[15,16]$. The D3 form is additionally produced in the skin on exposure to the sun $[15,16]$. Upon hydroxylation of pre-vitamin $\mathrm{D}$ in the liver, the main circulatory form of vitamin $\mathrm{D}$ (bound to albumin or in a free state), 25hydroxyvitamin D (25(OH)D) is produced [17]. Then, this $25(\mathrm{OH}) \mathrm{D}$ is converted into its active form, calcitriol $(1,25(\mathrm{OH}) 2 \mathrm{D})[15,18]$. Calcitriol stimulates intestinal calcium and phosphorus absorption and renal reabsorption of calcium via its receptors [16]. It also plays a role in the physiology of pregnancy by binding to the vitamin $\mathrm{D}$ receptors in the uteroplacental tissue $[15,18]$. The recommended dietary allowance and the tolerable upper level of vitamin D intake in pregnancy are 600 and 4000IU, respectively [15].

The trials on GDM patients have tested vitamin D antenatally in different dosages. As an oral supplement, while participants were advised to take $50,000 \mathrm{IU}$ of vitamin D at two to three weeks interval for about three to eight weeks in some trials $[13,14,19,20]$, other trials recommended 200-500IU of vitamin $\mathrm{D}$ daily $[21,22]$. The use of its injectable (intramuscular) forms was at a dosage of 300,000 IU [23]. Furthermore, while some trials have tested vitamin D as a sole prenatal supplement $[19,21,23]$, others have tested it as a cosupplement with other micronutrients like zinc, calcium, and magnesium [14,22].

Purpose of the proposed review

Parallel to the rising number of trials testing prenatal vitamin D supplementation's effect in GDM patients, systematic review and meta-analysis using data from these trials are also increasing. However, best known to this author, the burden and risk of attrition in these trials have not been systematically explored before [24]. Therefore, to study this poorly explored area of the GDM literature, a systematic review protocol is proposed here. The proposed review aims to determine the pooled weighted prevalence of missing outcome data among prenatal vitamin D receiving GDM patients and contrast its risk with the placebo recipients.

\section{Methods}

Studies matching the following eligibility criteria will be included in the proposed review.

\section{Inclusion criteria}

1. Study design: Parallel arm (any number of arms) randomized controlled trials, irrespective of their blindness and duration. 2 . Study population: Women of any age diagnosed with GDM during their concurrent pregnancy. Women will be recruited irrespective of their gravida, parity, or previous history of GDM diagnosis. 3. Intervention arm: A trial must test vitamin D as a sole or co-supplement with other nutrients in one or more of its treatment arms. 4. Comparator arm: The comparator arm should receive a placebo. 5. Outcome: Missing outcome data postrandomization will be the outcome of interest; however, it will not form the part of the inclusion criteria. Trialists' exclusion of any available outcome data from the statistical analysis will not be considered as missing outcome data. The diagnosis and management of GDM and the interventions' dosage, regimen, and administration route will be accepted as per the trialists.

\section{Exclusion criteria}

1. Any study design besides the above, like crossover trial or quasi-experimental study or observational study. 2. Women diagnosed with diabetes of non-GDM variants like diabetes type 1 or type 2 .

\section{Protocol registration}

This review protocol is registered with the PROSPERO (CRD42020180634) [25]. This reporting adheres closely to the reporting system commended by preferred reporting items for systematic review and meta-analysis protocols (PRISMA-P) 2015 statement [26].

\section{Database search}

As researchers and participants' safety is concerned, IRB should Electronic databases (PubMed, Embase, and Scopus) will be searched for the titles and abstracts of the English language publications irrespective of its publication date. A draft of the search strategy to be used in PubMed is presented here: "vitamin D" OR calciferol OR "vitamin D2" OR ergocalciferol 
OR "vitamin D3" OR cholecalciferol AND "gestational diabetes" OR GDM. Subsequent MeSH terms will be used in this search "cholecalciferol," "ergocalciferols," and "diabetes, gestational." The filters "Clinical Trial" and "Randomized Controlled Trial" will be used to narrow down the search results to RCTs. Additional searches will be done in the references of the reviewed trials.

\section{Study selection}

Citations retrieved from the database searches will be uploaded into Rayyan, a systematic review software [27]. Next, the duplicate references will be eliminated, and titles and abstracts of the remaining articles will be examined for eligible trials. Articles seeming to meet these criteria, or for those a decision about inclusion or exclusion into the proposed review cannot be made by reading these excerpts alone, a full-text reviewing will ensue. The last date of the database search, the count of citations requiring a full-text reading, and the reason for those eliminated after such reading will be maintained.

\section{Data extraction}

Data on the study design, population characteristics, compared interventions, and outcome of interest will be abstracted from each reviewed trial. In the study design, information on randomization, blinding, trial registration number, number of intervention arms, single or multi-centeredness, trial duration, nation where the trial was conducted, ethical clearance, participant consent, and funding information will be extracted. Subsequent participant details will be captured from each trial: the number of participants randomized to each of the intervention arms, their mean age, their diagnosis, and the criteria used to make the diagnosis of GDM (e.g., American Diabetic Association criteria) $[28,29]$. The content (e.g., vitamin D), dosage, regimen, and mode of administration of each intervention arm will be collected. The following details about the outcome will be abstracted from each intervention arm - the number of participants with missing outcome data and their reasons for missingness.

\section{Risk of bias assessment}

The Cochrane tool will be used to assess the selection, performance, detection, attrition, reporting, and other bias, and each of these biases will be categorized into a low, high, or unclear category [12]. The selection bias will be determined by the random sequence generation and its allocation concealment method from the participants [12]. To judge the performance and detection bias, the blinding mechanism of study personnel and participants and outcome assessors will be utilized, respectively [12]. The attrition bias evaluation will depend on the balance of attrition between the compared treatment arms and its reasons [12]. The trial results will be juxtaposed to its pre-specified intentions [12]. Other biases not categorizable into any of the above types will constitute a miscellaneous bias [12].

\section{Author role}

The review authors will independently select the eligible trials, abstract data, and assess the risk of bias. A third-party help will be sought when the authors fail to resolve any disagreement by discourse

\section{Analysis}

The overall and subgroup wise pooled weighted prevalence of missingness will be estimated in prenatal vitamin D supplemented GDM patients by the random-effect (DerSimonian and Laird) meta-analysis for binomial data using the exact binomial confidence interval determination method and Freeman-Tukey double arcsine transformation procedure for variance stabilization [30]. Subgrouping will be done by country and continent of the trial, and type of GDM treatment (between conservatively treated and medication treated).

The risk of missingness between antenatal vitamin $\mathrm{D}$ and placebo receiving GDM mothers will be contrasted by pairwise meta-analysis. Its modeling will depend on the presence (random-effect model using DerSimonian and Laird method) or absence (fixed-effect model utilizing the inverse-variance method) of clinical heterogeneity. The latter will be determined by the variations in the characteristics of the trials, participants, intervention providers, outcome assessors, and interventions. Trials without missing outcome data in both the contrasted treatment groups will not be incorporated in this pairwise metaanalysis. When this outcome does not occur in one of the trial's treatment arms, 0.5 will be added to each cell of the $2 \times 2$ table for meta-analysis. Trials with a high risk of bias component will be excluded from the meta-analysis to minimize bias in the summary estimates. The statistical significance will be determined at $\mathrm{p}<0.05$ and $95 \%$ confidence interval.

\section{Heterogeneity and publication bias}

For both binomial and pairwise meta-analysis, the heterogeneity will be assessed by $C h i^{2}$ (statistically significant at $P<0.1$ ) [12] and I2 (at values 25,50 , and $75 \%$, the heterogeneity will be categorized as low, moderate, and high, respectively) [31] statistics, and publication bias will be evaluated visually and statistically by funnel plots and Eggers test, respectively. Heterogeneity evaluation in the prevalence and pairwise metaanalysis will occur by meta-regression.

\section{Sensitivity analysis}

The meta-analyses will be repeated while eliminating a trial each time to evaluate the preliminary meta-analysis's robustness. Besides, the pairwise meta-analysis will be iterated using a model (random-effect or fixed-effect) alternative to that used in the primary analysis. All analyses will be done in Stata statistical software, version 16 (StataCorp, College Station, Texas, USA). A statistically significant estimate of the pairwise meta-analysis will undergo a quality assessment with the Grading of Recommendations Assessment, Development and Evaluation (GRADE) approach (by GRADE Working Group (2004)) [32]. If the quantitative analysis is not possible, narrative reporting will follow. The review's reporting will adhere to preferred reporting items for systematic reviews and meta-analyses guidelines (PRISMA) [33].

\section{Discussion}

Contemporarily, several trials have tested the effects of antenatal vitamin D supplementation on GDM mothers and their infants; however, it is not clear if missing outcome data threatens these trials' internal validity meta-analyses that abstract data from these. Therefore, a systematic review protocol is proposed here to determine the prevalence of 
missing outcome data in vitamin D supplemented GDM mothers and compare its risk with placebo recipients.

The strengths of the proposed review will perhaps be the first systematic review and meta-analysis to estimate the epidemiologic burden of missingness among vitamin D receiving GDM mothers and compare its risk with placebo recipients. Besides, the evidence generated from it is likely to be rigorous as it will be based on RCTs, the highest level of the epidemiologic evidence. Furthermore, the database search's non-restrictiveness to any date or geographical boundary will perhaps ensure the proposed review. Concerning the weaknesses of the prospective review, it is expected to suffer from the following limitations. If the trials included in the proposed review primarily uses vitamin D with co-supplements, extricating vitamin D's effect from the latter will be difficult. Likewise, accepting the GDM treatment as per the trialists may cause the recruitment of a substantial number of trials using insulin and/or oral hypoglycemics to treat GDM. In such a situation, disentangling its effect on the outcome from that of vitamin D supplements' might be challenging. Lastly, the inclusion of articles published in the English language only will decrease the possibility of identifying relevant trials (if any) published in any other language. Despite these limitations, this review will be one of the preliminary sources of evidence to provide knowledge on the epidemiologic burden and risk of missing outcome data on trials that tested vitamin D as a supplement in GDM mothers and guide future meta-analysts to understand the internal validity of these trials better. Simultaneously, the evidence might prove useful to future trialists in developing novel trials in terms of minimizing attrition and retaining internal validity.

\section{Conclusion}

The proposed review will estimate the prevalence of missing outcome data in RCTs testing the effects of vitamin D supplementation in GDM mothers and juxtapose its risk with placebo recipients.

\section{Abbreviation}

GDM: Gestational Diabetes Mellitus; RCT: Randomized Controlled Trials; 25(OH)D: 25-hydroxyvitamin D; 1,25(OH)2D: Calcitriol; PRISMA-P: Preferred Reporting Items for Systematic Review and Meta-Analysis Protocols; GRADE: Grading of Recommendations Assessment, Development, and Evaluation; PRISMA: Preferred Reporting Items for Systematic reviews and Meta-Analyses guidelines; IU: International Unit

\section{Funding}

The author received no financial support for the research, authorship, and/or publication of this article.

\section{Availability of data and materials}

Data will be available by emailing sumanta.saha@uq.net.au

\section{Authors' contributions}

Sumanta Saha (SS) is the principal investigator of this manuscript (systematic review protocol). SS is responsible for the study concept, design, writing, reviewing, editing, and approving the manuscript in its final form. SS read and approved the final manuscript.
Ethics approval and consent to participate

We conducted the research following the Declaration of Helsinki. However, the systematic review protocol article needs no ethics committee approval.

\section{Consent for publication}

Not applicable

\section{Competing interest}

The author declares that he has no competing interests.

\section{Open Access}

This article is distributed under the terms of the Creative Commons Attribution 4.0 International License (http://creativecommons.org/licenses/by/4.0/), which permits unrestricted use, distribution, and reproduction in any medium, provided you give appropriate credit to the original author(s) and the source, provide a link to the Creative Commons license, and indicate if changes were made. The Creative Commons Public Domain Dedication waiver (http://creativecommons.org/publicdomain/zero/1.0/) applies to the data made available in this article, unless otherwise stated.

Author details

${ }^{1}$ Independent Researcher, India. ORCID ID: 0000-0003-09968846

\section{Article Info}

Received: 02 September 2020

Accepted: 09 October 2020

Published: 22 October 2020

References

1. Quintanilla Rodriguez BS, Mahdy H. Gestational Diabetes. [Updated 2019 Dec 23]. StatPearls. Treasure Isl StatPearls Publ. 2020. Available from: https://www.ncbi.nlm.nih.gov/books/NBK545196/

2. Committee on Practice Bulletins-Obstetrics. ACOG Practice Bulletin No. 190: Gestational Diabetes Mellitus. Obstet Gynecol. 2018;131: e49-64. Available from: http://www.ncbi.nlm.nih.gov/pubmed/29370047

3. Mack LR, Tomich PG. Gestational Diabetes: Diagnosis, Classification, and Clinical Care. Obstet Gynecol Clin North Am [Internet]. 2017; 44:207-17. Available from: http://www.ncbi.nlm.nih.gov/pubmed/28499531

4. Coustan DR. Gestational Diabetes Mellitus. Clin Chem. 2013; 59:1310-21. Available from: http://www.clinchem.org/cgi/doi/10.1373/clinchem.2013.203331

5. 14. Management of Diabetes in Pregnancy: Standards of Medical Care in Diabetes-2019. Diabetes Care. 2019;42: S165-72. Available from: http://care.diabetesjournals.org/lookup/doi/10.2337/dc19-S014

6. Saha S. Compliance and barriers to self-monitoring of blood glucose in patients with gestational diabetes mellitus: A systematic review. Int J Health Sci (Qassim).2019;13:44-52. Available from: http://www.ncbi.nlm.nih.gov/pubmed/31123440

7. Akbari M, Mosazadeh M, Lankarani K, Tabrizi R, Samimi M, Karamali M, et al. The effects of vitamin d supplementation on glucose metabolism and lipid profiles in patients with gestational diabetes: a systematic review and meta-analysis of randomized controlled trials. Horm Metab Res.Germany; 2017;49:647-53. Available from: http://www.thiemeconnect.de/DOI/DOI?10.1055/s-0043-115225 
8. Saha S, Saha S. A comparison of the risk of cesarean section in gestational diabetes mellitus patients supplemented antenatally with vitamin D containing supplements versus placebo: A systematic review and meta-analysis of double-blinded randomized controlled trials. J Turkish-German Gynecol Assoc. 2020

21:201-12. https://dx.doi.org/10.4274\%2Fjtgga.galenos.2020.2019.0164

9. Saha SS, Saha SS. The risk of morbidities in newborns of antenatal vitamin D supplemented gestational diabetes mellitus patients. Int J Health Sci (Qassim). Qassim University; 2020; 14:3-17. Available from https://www.ncbi.nlm.nih.gov/pmc/articles/PMC7475207/

10. Saha S, Saha S. A Comparison of the Changes in Gestational Weight, Body Mass Index, and Serum Vitamin D Level in Gestational Diabetes Mellitus Patients Complemented with Vitamin D in Contrast to Those Who Did Not Receive the Supplement: A Protocol for Systematic Review a. Int J Diabetes Metab. S. Karger AG; 2019; 25:74-9. https://doi.org/10.1159/000505269

11. Mavridis D, White IR. Dealing with missing outcome data in meta-analysis. Res Synth Methods.2020;11:2-13. Available from: https://onlinelibrary.wiley.com/doi/abs/10.1002/jrsm.1349

12. Higgins JPT GS (editors). Cochrane Handbook for Systematic Reviews of Interventions Version 5.1.0 [updated March 2011]. Cochrane Collab. 2011 [cited 2020 Aug 27]. Available from: https://training.cochrane.org/handbook/archive/v5.1/

13. Asemi Z, Hashemi T, Karamali M, Samimi M, Esmaillzadeh A Effects of vitamin D supplementation on glucose metabolism, lipid concentrations, inflammation, and oxidative stress in gestational diabetes: a double-blind, randomized controlled clinical trial. Am J Clin Nutr.2013;98:1425-32. https://doi.org/10.3945/ajen.113.072785

14. Asemi Z, Karamali M, Esmaillzadeh A. Effects of calciumvitamin D co-supplementation on glycaemic control, inflammation and oxidative stress in gestational diabetes: a randomised placebocontrolled trial. Diabetologia .2014;57:1798-806. https://doi.org/10.1007/s00125-014-3293-x

15. Institute of Medicine (US) Committee to Review Dietary Reference Intakes for Vitamin D and Calcium. Dietary Reference Intakes for Calcium and Vitamin D. Ross AC, Taylor CL, Yaktine AL, Del Valle HB, editors. Washington (DC): National Academies Press (US); 2011. Available from: https://books.google.co.in/books?id=VF2aRQJ6IZ4C

16. Gossman W, Chauhan K, Huecker MR. Vitamin D. StatPearls. 2019. Available from: http://www.ncbi.nlm.nih.gov/pubmed/28722941

17. Curtis EM, Moon RJ, Harvey NC, Cooper C. Maternal vitamin D supplementation during pregnancy. Br Med Bull. 2018; 126:5777. https://doi.org/10.1093/bmb/ldy010

18. Knabl J, Vattai A, Ye Y, Jueckstock J, Hutter S, Kainer F, et al. Role of Placental VDR Expression and Function in Common Late Pregnancy Disorders. I Int J Mol Sci. 2017;18(11):2340. https://doi.org/10.3390/ijms18112340

19. Yazdchi R, Gargari BP, Asghari-Jafarabadi M, Sahhaf F. Effects of vitamin D supplementation on metabolic indices and hs-CRP levels in gestational diabetes mellitus patients: a randomized, double-blinded, placebo-controlled clinical trial. Nutr Res Pract. 2016; 10:328. Available from: http://synapse.koreamed.org/DOIx.php?id=10.4162/nrp.2016.10.3 .328

20. Jamilian M, Samimi M, Ebrahimi FA, Hashemi T, Taghizadeh M, Razavi M, et al. The effects of vitamin D and omega-3 fatty acid co-supplementation on glycemic control and lipid concentrations in patients with gestational diabetes. J Clin Lipidol. 2017; 11:45968. https://doi.org/10.1016/j.jacl.2017.01.011
21. Li Q, Xing B. Vitamin D3-Supplemented Yogurt Drink Improves Insulin Resistance and Lipid Profiles in Women with Gestational Diabetes Mellitus: A Randomized Double Blinded Clinical Trial. Ann Nutr Metab. 2016; 68:285-90. https://doi.org/10.1159/000447433

22. Karamali M, Bahramimoghadam S, Sharifzadeh F, Asemi Z Magnesium-zinc-calcium-vitamin D co-supplementation improves glycemic control and markers of cardiometabolic risk in gestational diabetes: a randomized, double-blind, placebocontrolled trial. Appl Physiol Nutr Metab. 2018; 43:565-70. Available

from: http://www.nrcresearchpress.com/doi/10.1139/apnm-2017-0521

23. Hosseinzadeh-Shamsi-Anar M, Mozaffari-Khosravi H, Salami MA, Hadinedoushan H, Mozayan MR. The efficacy and safety of a high dose of vitamin $\mathrm{d}$ in mothers with gestational diabetes mellitus: a randomized controlled clinical trial. Iran J Med Sci. 2012; 37:159-65. Available from: http://www.ncbi.nlm.nih.gov/pubmed/23115447

24. Saha S. Impact of missingness on clinical trials on the effectiveness of antenatal vitamin D supplementation in gestational diabetes mellitus patients. J Ideas Heal. 2020; 3:138-9. https://doi.org/10.47108/jidhealth.Vol3.Iss1.47

25. Saha S, Saha S. The variation in participant attrition between prenatal vitamin D supplemented and not supplemented gestational diabetes mellitus patients: a systematic review and meta-analysis of randomized controlled trials. PROSPERO. 2020. https://www.crd.york.ac.uk/prospero/display_record.php?ID=CR D42020180634

26. Moher D, Shamseer L, Clarke M, Ghersi D, Liberati A, Petticrew $\mathrm{M}$, et al. Preferred reporting items for systematic review and metaanalysis protocols (PRISMA-P) 2015 statement. Syst Rev. 2015; 4:1. https://doi.org/10.1186/2046-4053-4-1

27. Ouzzani M, Hammady H, Fedorowicz Z, Elmagarmid A. Rayyan - a web and mobile app for systematic reviews. Syst Rev. 2016; 5:210. https://doi.org/10.1186/s13643-016-0384-4

28. American Diabetes Association. Diagnosis and classification of diabetes mellitus. Diabetes Care. 2014;37 Suppl 1: S81-90. https://doi.org/10.2337/dc14-s081

29. Rossi G, American Diabetes Association. [Diagnosis and classification of diabetes mellitus]. Recenti Prog Med. 2010; 101:274-6. http://www.ncbi.nlm.nih.gov/pubmed/20842952

30. Nyaga VN, Arbyn M, Aerts M. Metaprop: a Stata command to perform meta-analysis of binomial data. Arch Public Heal. 2014; $72: 39$

http://archpublichealth.biomedcentral.com/articles/10.1186/2049 3258-72-39

31. Higgins JPT, Thompson SG, Deeks JJ, Altman DG. Measuring inconsistency in meta-analyses. BMJ. 2003; 327:557-60. http://www.bmj.com/cgi/doi/10.1136/bmj.327.7414.557

32. Atkins D, Best D, Briss PA, Eccles M, Falck-Ytter Y, Flottorp S, et al. Grading quality of evidence and strength of recommendations. $\quad$ BMJ. 2004; 328:1490. http://www.bmj.com/lookup/doi/10.1136/bmj.328.7454.1490

33. Liberati A, Altman DG, Tetzlaff J, Mulrow C, Gøtzsche PC, Ioannidis JPA, et al. The PRISMA statement for reporting systematic reviews and meta-analyses of studies that evaluate health care interventions: explanation and elaboration. J Clin Epidemiol.2009;62:

e1-34 https://doi.org/10.1016/j.jclinepi.2009.06.006 